\title{
Electroencephalographic dynamics of etomidate-induced loss of consciousness
}

\author{
Lei Zhang ${ }^{1,3}$, Shunqin Fan²,3, Jiawei Zhang ${ }^{2,3}$, Kun Fang ${ }^{2,3}$, Lei Wang ${ }^{1,3}$, Yuanyuan Cao ${ }^{1,3}$, Lijian Chen ${ }^{1,3}$, \\ Xuesheng Liu ${ }^{1,3}$ and Erwei Gu, ${ }^{1,3^{*}}$
}

\begin{abstract}
Background: Highly structured electroencephalography (EEG) oscillations can occur in adults during etomidateinduced general anesthesia, but the link between these two phenomena is poorly understood. Therefore, in the present study, we investigated the electroencephalogram dynamics of etomidate-induced loss of consciousness (LOC) in order to understand the neurological mechanism of etomidate-induced LOC.
\end{abstract}

Methods: This study is a prospective observational study. Etomidate-induced anesthesia was performed on eligible patients undergoing elective surgery. We analyzed EEG data from 20 patients who received etomidate for the induction of general anesthesia. We used power spectra and coherence methods to process and analyze the EEG data. Our study was based on 4-channel EEG recordings.

Results: Compared with the baseline (awake period), etomidate induced an increase in power in delta, theta, alpha and beta waves during LOC. Compared with the awake period, the delta-wave $(1-4 \mathrm{~Hz})$, alpha-wave $(8-13 \mathrm{~Hz})$, and theta-wave $(4-8 \mathrm{~Hz})$ coherence increased significantly during LOC, while the slow-wave $(<1 \mathrm{~Hz})$ coherence decreased. However, the delta wave $(1.0-4.0 \mathrm{~Hz})$ during etomidate-induced LOC was more coherent than during the awake period $(1.86-3.17 \mathrm{~Hz}$, two-group test for coherence, $p<0.001)$.

Conclusions: The neural circuit mechanism of etomidate-induced LOC is closely related to the induction of oscillation in delta, theta, alpha and beta waves and the enhancement of delta-wave coherence.

Trial registration: ChiCTR1800017110

Keywords: EEG, Etomidate, LOC, General anesthesia

\section{Background}

Etomidate is a non-barbiturate intravenous anesthetic that has a rapid onset of action and induces a stable, quiet, comfortable, and non-excitable transition during the induction period. Enhancement of $\gamma$-aminobutyric acid A receptor (GABAAR) activity is considered to be the primary mechanism mediating etomidate-induced anesthesia [1, 2]. Etomidate has little effect on the

\footnotetext{
*Correspondence: ay_guew_mz@163.com

'Department of Anesthesiology, The First Affiliated Hospital of Anhui Medical University, No. 218 Jixi road, Anhui province 230022 Hefei, China

${ }^{3}$ Key Laboratory of Anesthesiology and Perioperative Medicine of Anhui Higher Education Institutes, Anhui Medical University, Hefei, China Full list of author information is available at the end of the article
}

cardiovascular and respiratory systems. Based on these characteristics, etomidate is widely used in the induction of anesthesia in patients with impaired hemodynamics, such as in the elderly and those with cardiovascular disease or critical illness [3, 4]. General anesthetics are well described at the molecular level and cellular pharmacological level, but the neural circuit mechanisms that cause LOC remain unclear $[5,6]$. Electroencephalography (EEG) is considered to be the most direct indicator of central nervous system activity [7, 8]. The quantitative evaluation of the depth of anesthesia by EEG has made an important contribution to the practice of clinical anesthesia [7]. A recent study reported that in

(c) The Author(s). 2021 Open Access This article is licensed under a Creative Commons Attribution 4.0 International License, which permits use, sharing, adaptation, distribution and reproduction in any medium or format, as long as you give appropriate credit to the original author(s) and the source, provide a link to the Creative Commons licence, and indicate if changes were made. The images or other third party material in this article are included in the article's Creative Commons licence, unless indicated otherwise in a credit line to the material. If material is not included in the article's Creative Commons licence and your intended use is not permitted by statutory regulation or exceeds the permitted use, you will need to obtain permission directly from the copyright holder. To view a copy of this licence, visit http://creativecommons.org/licenses/by/4.0/ The Creative Commons Public Domain Dedication waiver (http://creativecommons.org/publicdomain/zero/1.0/) applies to the data made available in this article, unless otherwise stated in a credit line to the data. 
general anesthesia, the effect of propofol on neuronal data recorded in the frontal cortex, and the study found that frontal-lobe electrical activity is the precursor to a LOC [9].

Etomidate-induced general anesthesia produces dynamic changes in EEG data[10], but the characteristics of these changes have not been well elucidated. Furthermore, the relationship between etomidateinduced LOC and characteristic changes in EEG data is not well understood. Kuizenga et al.[10] studied the amplitude of the specified band, spectral edge frequency $95 \%$ (SEF95) and bispectral index (BIS), to explore the relationship between EEG effects and the moment of LOC.Our research mainly used spectral and coherence analysis to clarify the relationship between etomidate-induced LOC and the characteristic changes in EEG dynamics.

\section{Methods}

This study followed the Declaration of Helsinki and was approved by the Ethics Committee of the First Affiliated Hospital of Anhui Medical University. Standard monitoring techniques (noninvasive blood pressure, electrocardiography [ECG], and pulse oximetry) were applied. Hemodynamic variables were recorded every $60 \mathrm{~s}$. Written informed consent was obtained from the included patients, who were between 18 and 65 years old (American Society of Anesthesiologists (ASA): class I to II; No gender limit) and required general anesthesia. Exclusion criteria were as follows: pregnancy, hearing impairments, mental disorders, or taking drugs that may interfere with the accuracy of EEG recordings. Myoclonus often occurs during the induction of etomidate anesthesia [11, 12]. The patient's EEG data was not included in the data analysis if myoclonus occurred clinically.

We used a four-channel Sedline brain function monitor (Masimo,Irvine, CA,USA) to record the frontal-lobe EEG data. EEG data was recorded in patients undergoing elective surgery from a baseline of $3 \mathrm{~min}$ (awake and quiet with their eyes closed) to $3 \mathrm{~min}$ of etomidateinduced LOC. Etomidate $(0.06 \mathrm{mg} / \mathrm{kg} / \mathrm{min})$ was the only anesthetic used [10]. The EEG data was recorded with a preamplifier bandwidth of $0.5-92 \mathrm{~Hz}$, a sampling rate of $178 \mathrm{~Hz}, 16$ bits. The standard Sedline-Sedtrace electrode array records were from electrodes located roughly at positions Fp1, Fp2, F7, and F8, with the ground electrode at Fpz and the reference electrode at roughly $1 \mathrm{~cm}$ above Fpz (Fig. 1). The electrode impedance was less than $5 \mathrm{k} \Omega$ in each channel. The time recorded in the case report form was required to match the time on the EEG recorder in order to mark key events (e.g., induction start, LOC) during the analysis.

\section{Criteria for the LOC}

At present, in the induction of clinical anesthesia, the determination of the LOC is assessed by aimless movements after harmful stimulation [13]. In the present study, the auditory stimulation assessment $[14,15]$ was supplemented by the disappearance of the eyelash reflex to confirm the LOC. Before inducing anesthesia, we instructed patients not to open or move their eyes.

\section{Data preprocessing}

A researcher with experience in reading electroencephalograms manually browsed the EEG data of each patient to manually remove artifacts. The investigator used the recorded information in the case report form to select the appropriately timed EEG data segment. For each case, the EEG segment representing $60 \mathrm{~s}$ of consciousness and closed eyes was carefully selected during the perioperative period, as was the EEG segment corresponding to $60 \mathrm{~s}$ after the LOC, for data analysis.

\section{Spectral analysis}

The power spectrum quantifies the energy of each frequency of the EEG. The spectrogram was computed using the multitaper method achieved in the Chronux toolbox in MATLAB[16]. The group-level spectrogram computed by taking the median of spectrograms of all patients (Fig. $2 \mathrm{a} / \mathrm{b}$ ). The spectrum of the selected EEG epochs (EEG epoching is a procedure in which specific time-windows are extracted from the continuous EEG signal) was also calculated by us. Then, for all epochs, the resulting power spectra was averaged, and by way of multitaper-based jackknife techniques [16], $95 \%$ confidence intervals (CIs) were computed. Parameters for spectral analysis are as follows: window length $\mathrm{T}=2 \mathrm{~s}$ with a $1.95 \mathrm{~s}$ overlap; time-bandwidth product of $\mathrm{TW}=$ 3 (Approximately 2WT Slepian functions fit on this Time-Frequency tile. Since T, W are input parameters, we can easily control the resolution element in the Time Frequency plane using Slepians); number of tapers, $K=$ 5; and spectral resolution $=3 \mathrm{~Hz}$ (Fig. 3).

\section{Coherence analysis}

Coherence graphs are coherent time-varying versions, which are estimated using continuous windows of EEG data. Between two signals, $x$ and $y$, the coherence Cxy (f) function, is determined as follows:

$$
\operatorname{Cxy}(f)=\frac{|\operatorname{Sxy}(f)|}{\sqrt{ } \operatorname{Sxx}(f) \operatorname{Syy}(f)},
$$

Sxy (f) is the cross-spectrum between the signals $x(t)$ and $y(t), \operatorname{Sxx}(f)$ is the power spectrum of the signal $x$ $(\mathrm{t})$, and Syy (f) is the power spectrum of the signal y (t) [16]. In order to acquire the appraised coherence, based 


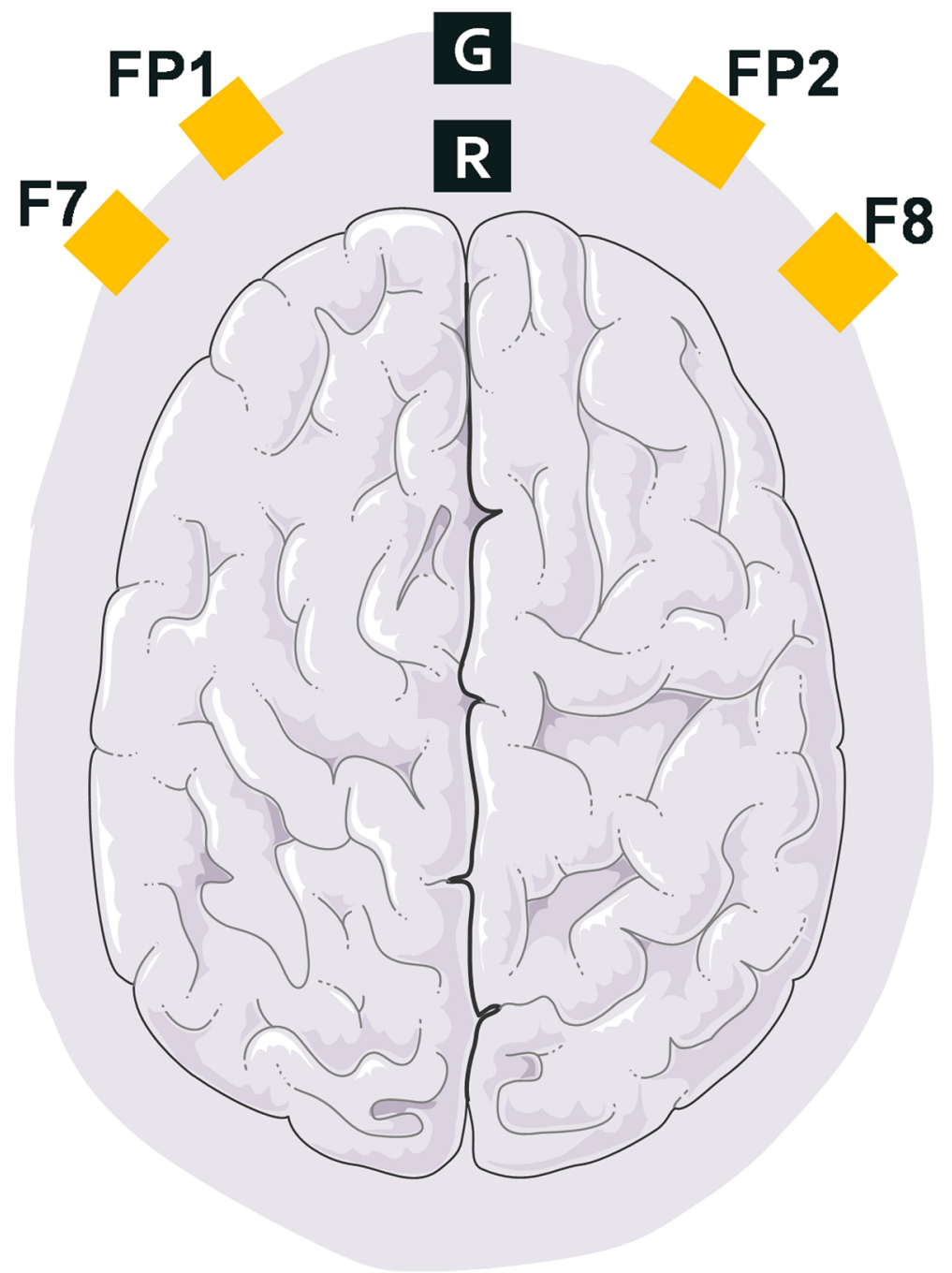

Fig. 1 The channel position and the two bipolar frontal channels: F7 and F8, which we used for coherence analysis

on the Chronux toolbox in MATLAB, the coherence was computed between F7 and F8, the two frontal electrodes[17]. By taking the median across subjects, the group-level coherograms were computed. The coherence for selected EEG epochs was also calculated. The resulting coherence estimates were averaged for all epochs, and by way of multitaper-based jackknife techniques, $95 \%$ CIs were computed [16]. Parameters for the coherence analysis were similar to spectral analysis and spectral resolution of $2 \mathrm{~W}=3 \mathrm{~Hz}$.

\section{Statistical analysis}

So as to compare spectral and coherence estimates between groups, we utilized jackknife-based methods [16], the two-group test for spectra, and the two-group test for coherence, as performed in the Chronux toolbox routine [18]. This method takes into account the frequency spectrum and the basic spectral resolution of the coherence estimation, and only when the difference occurs at a continuous frequency on a frequency band wider than the spectral resolution of $2 \mathrm{~W}$, the difference is considered significant. To be specific, for frequencies $\mathrm{f}>2 \mathrm{~W}$, the negative assumption was rejected only if the test statistic surpassed the significance threshold over a contiguous frequency range $\geq 2 \mathrm{~W}$. For frequencies $0 \leq$ $\mathrm{f} \leq 2 \mathrm{~W}$, in order to illustrate the capabilities of multitaper spectral estimation when the frequency is near zero, the negative assumption was rejected only if the test statistic surpassed the significance threshold over a contiguous frequency range from 0 to $\max (\mathrm{f}, \mathrm{W}) \leq 2 \mathrm{~W}$. A significance threshold of $p<0.001$ was confirmed for comparisons between groups. 

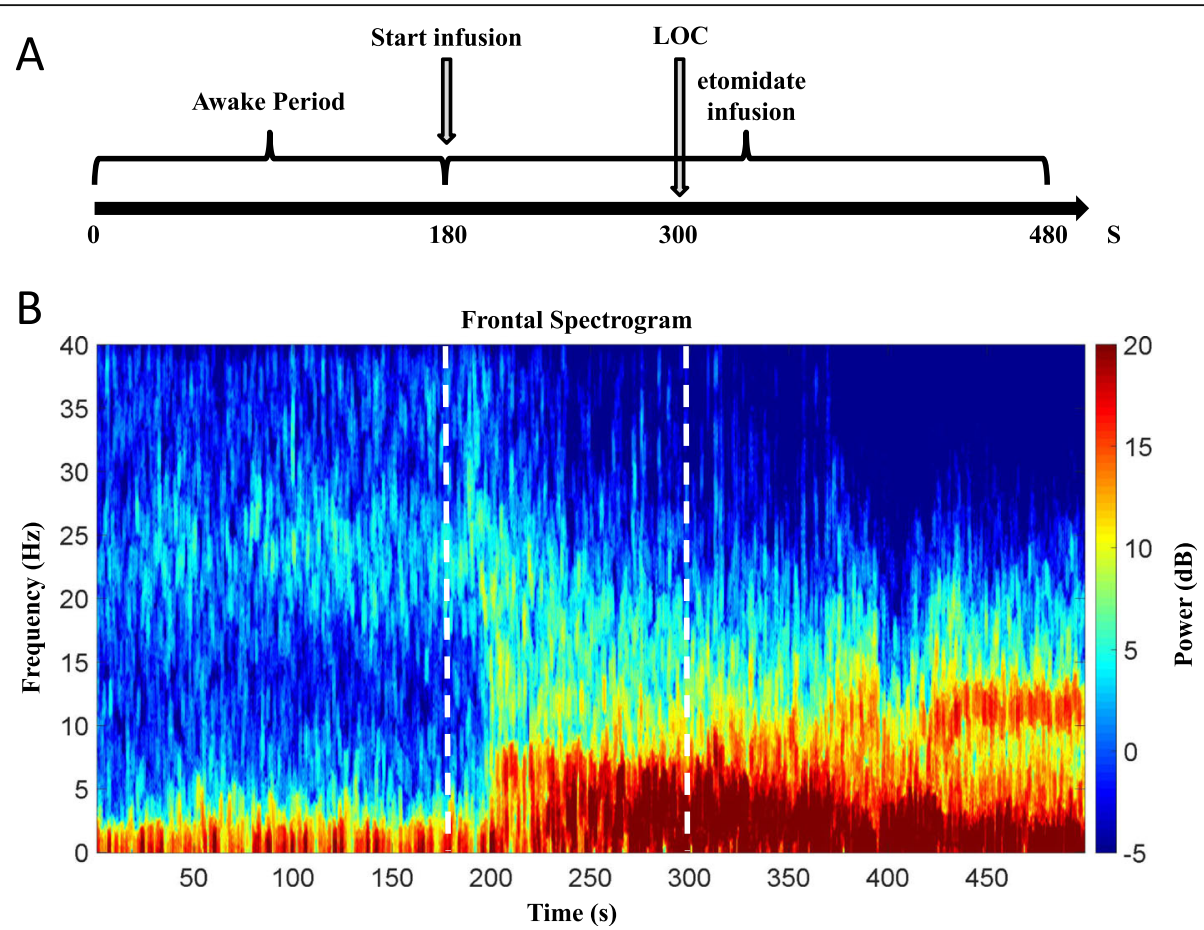

Fig. 2 Time-frequency analysis of EEG data between the awake period and etomidate-induced LOC. a During the awake period before induction of etomidate $(n=20)$, slow-wave $(<1.0 \mathrm{~Hz})$ and delta-wave $(1.0-4.0 \mathrm{~Hz})$ oscillations were mainly present. b Etomidate-induced LOC $(n=20)$ yielded an increase in the power of slow waves $(<1.0 \mathrm{~Hz})$, delta waves $(1.0-4.0 \mathrm{~Hz})$, theta waves $(4.0-8.0 \mathrm{~Hz})$, and alpha waves $(8.0-13.0 \mathrm{~Hz})$. c Spectrograms of the awake period. Compared with those during the awake period, the powers of the slow wave $(<1.0 \mathrm{~Hz})$, delta wave $(1.0-$ $4.0 \mathrm{~Hz})$, theta wave $(4.0-8.0 \mathrm{~Hz})$, and alpha wave $(8.0-13.0 \mathrm{~Hz})$ during the etomidate-induced LOC were significantly increased $(\mathrm{C}: 0-22.97 \mathrm{~Hz}$, 27.28-40.00 Hz; $p<0.001$, two-group test for spectra). Median spectra presented with $95 \%$ jackknife Cls. Horizontal solid black lines represent the frequency ranges at which there were significant differences

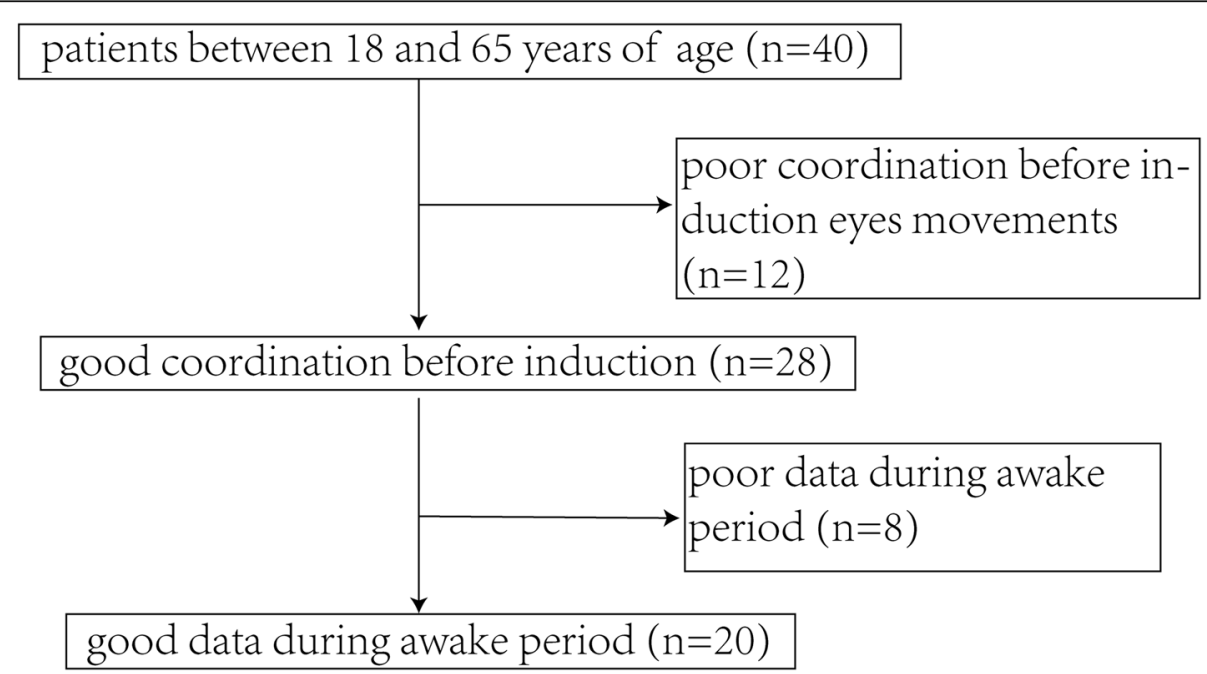

Fig. 3 Frontal-lobe EEG spectrograms in the study of etomidate-induced LOC. a In the etomidate study, the main events are marked on the timeline. $\mathbf{b}$ During the study, each patient was instructed to keep their eyes closed and without motion for a period of 3 min before etomidate was administered to induce general anesthesia. The frequency is plotted on the $y$-axis and time is plotted on the $x$-axis. The energy or power in the signal is represented by color. As the induction of etomidate began, the slow-wave, delta-wave, and theta-wave oscillations increased. After LOC, the alpha wave oscillation increased 

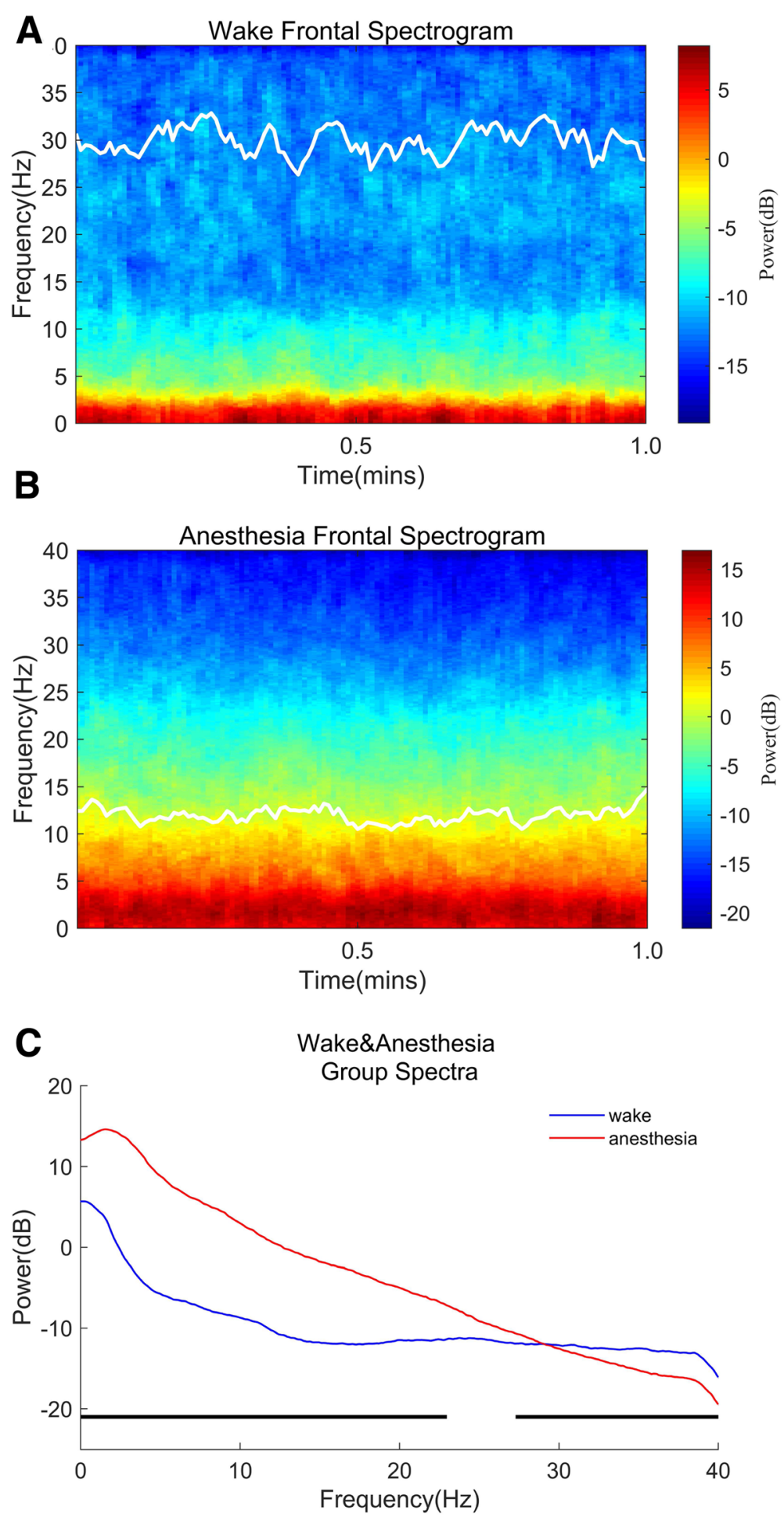

Fig. 4 Case selection. Our study initially included 40 individuals between 18 and 65 years old. We excluded 12 patients because of restlessness or other non-cooperative behaviors before anesthesia. We then examined the EEG data of the remaining 28 patients and excluded 8 patients due to poor data quality (Poor electrode contact and other reasons). Ultimately, we analyzed the EEG data from 20 patients 


\section{Results}

\section{Case selection}

Our study initially included 40 individuals between 18 and 65 years old. Of these, we excluded patients who had poor cooperation before anesthesia $(n=12)$. Then we imported the EEG data of the remaining patients $(n=28)$ into EEGLAB and excluded patients $(n=8)$ due to poor electrode contact (Fig. 4). Table 1 shows the basic demographic and clinical characteristics of the included cases.

\section{Etomidate power-spectra analysis}

We observed the EEG spectra during both the awake period before etomidate administration and during etomidate-induced LOC. The two time-frequency diagrams were continuous in time, and the EEG power changed significantly over time. The awake period and the period corresponding to the etomidate-induced LOC were dominated by slow-wave $(0.1-1.0 \mathrm{~Hz})$ and deltawave $(1.0-4.0 \mathrm{~Hz})$ oscillations. And the period of LOC also showed the theta-wave $(4.0-8.0 \mathrm{~Hz})$ and alpha-wave (8.0-13.0 Hz) oscillations. Next, we performed timefrequency analysis of EEG data in the two periods and found that after etomidate-induced LOC, the oscillation in delta, theta, alpha and beta waves increased. We also observed that the two periods were clearly different in time and frequency between 0 and $22.97 \mathrm{~Hz}$ and 27.28$40.00 \mathrm{~Hz}$ (Fig. 2).

\section{Etomidate coherence analysis}

We next analyzed the similarities and differences in the correlation patterns between the awake period and the period during etomidate-induced LOC. The period of awake showed the coherence spectrum in terms of the slow wave $(<1.0 \mathrm{~Hz})$, delta wave $(1.0-4.0 \mathrm{~Hz})$, and alpha wave $(8.0-13.0 \mathrm{~Hz})$. The period of etomidateinduced LOC shows the coherence spectra in terms of the delta wave $(1.0-4.0 \mathrm{~Hz})$, theta wave $(4.0-8.0 \mathrm{~Hz})$, and the alpha wave $(8.0-13.0 \mathrm{~Hz})$. During the awake period, the coherence of slow waves $(<1.0 \mathrm{~Hz})$ was decreased. We also observed that there was a significant difference in the coherence between the waking period and the period of etomidate-induced LOC in the 1.863.17 $\mathrm{Hz}$ band (Fig. 5).

Table 1 Basic features information of case objects

\begin{tabular}{ll}
\hline & Etomidate $(\boldsymbol{n}=\mathbf{2 0})$ \\
\hline Sex $($ male/\%) & $8(40)$ \\
Weight $(\mathrm{kg})$, mean $( \pm \mathrm{SD})$ & $36(10)$ \\
Height $(\mathrm{cm})$, mean $( \pm \mathrm{SD})$ & $61(9)$ \\
Time of LOC $(\mathrm{s})$, mean $( \pm \mathrm{SD})$ & $164(7)$ \\
\hline
\end{tabular}

\section{Discussion}

In the present study, we analyzed the characteristic changes in the EEG bands before and during etomidateinduced anesthesia via spectral analysis and coherence analysis. From the awake period to the LOC period, etomidate general anesthesia induced an increase in oscillation in each frequency band within the EEG data. Additionally, the coherence of the EEG signal in the theta wave and alpha wave was obviously enhanced, when etomidate induced LOC; and the delta wave also showed obvious coherence.

Etomidate exerts its anesthetic actions through potentiation of GABAARs containing $\beta 2$ and $\beta 3$ subunits. It has recently been shown that the $\beta 2$ subunit contributes to the sedative properties of etomidate, whereas the 33 subunit is responsible for its anesthetic properties $[19,20]$. In our present study, etomidate induced anesthesia and enhanced theta-wave oscillations. In our coherence analysis, etomidate produced strong coherence in the theta wave; interestingly, $\beta 3$-subunitcontaining receptors play an important role in thetawave oscillations. A previous study analyzed the effects of etomidate on rhythmic population activity by recording local field potentials (LFPs) [21]. In slices, which are derived from wild-type mice, etomidate (200 nM) amplified the oscillatory population activity in the thetafrequency band, but this effect was not seen in slices, which derived from $\beta 3$-knockin mice, and this phenomenon was also observed in vivo [22]. These findings indicate that the neuronal mechanism of etomidateinduced LOC is closely related to the $\beta 3$ subunit of GABAAR functional activity. Furthermore, previous evidence has shown that the overall effect of etomidate reflects a balance between enhancement and inhibition produced by GABAARs containing $\beta 2$ and $\beta 3$ subunits [21]. Etomidate enhances theta oscillations by acting on $\beta 3$-containing GABAA receptors but depresses these oscillations via $\beta 2$-subunit-containing receptors. In our present study of etomidate-induced LOC, we systematically observed the enhancement of EEG theta oscillations. However, which receptors participate and play a role is not known in our research, and further observation and research are needed.

In the present study, etomidate induced LOC and changed the EEG theta rhythm. Some studies have indicated that hippocampal interactions with the prefrontal cortex, another significant memory-associated structure, are coordinated by theta-rhythm plasticity [23, 24]. Amnesia is a significant symptom of general anesthesia. Several different brain areas participate in memory formation, including the prefrontal cortex, amygdala, and hippocampus. Anesthesia may be associated with memory impairment, as has been indicated by many studies in the hippocampus $[25,26]$, and evidence suggests that 


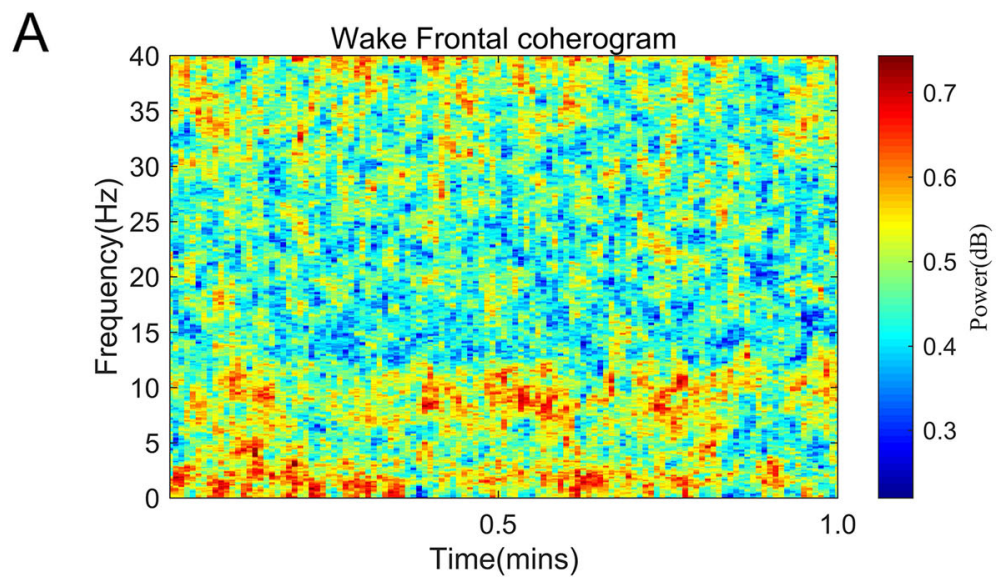

B

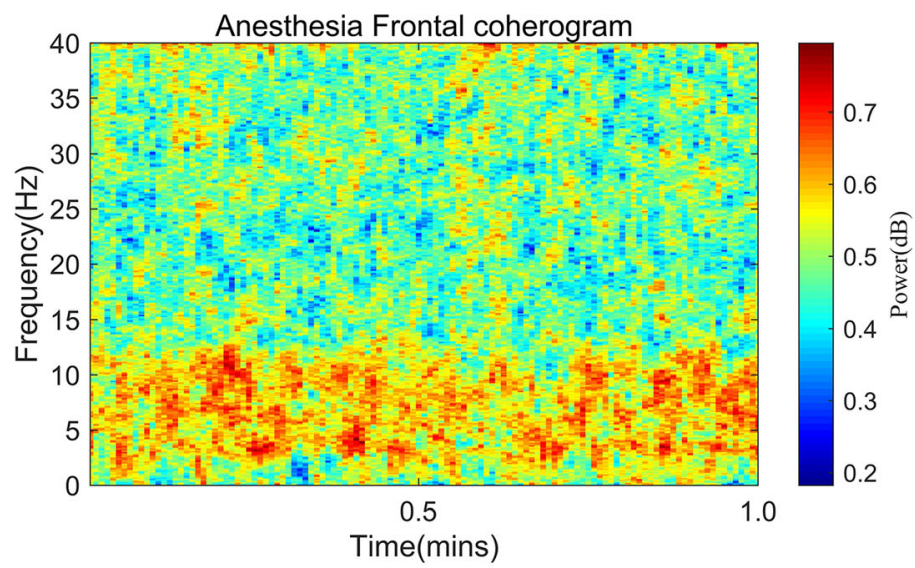

C

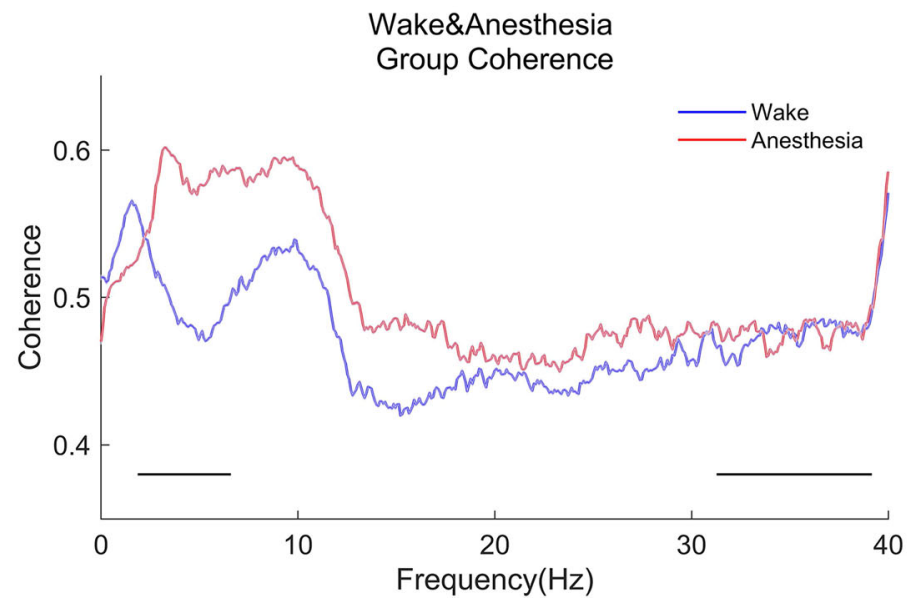

Fig. 5 Coherence analysis between the awake period and the period during etomidate-induced LOC. a The coherence analysis of the awake period $(n=20)$ showed the coherence spectrum in terms of the slow wave $(<1.0 \mathrm{~Hz})$, delta wave $(1.0-4.0 \mathrm{~Hz})$, and alpha wave $(8.0-13.0 \mathrm{~Hz})$. b The coherence analysis during etomidate-induced LOC $(n=20)$ shows the coherence spectra in terms of the delta wave $(1.0-4.0 \mathrm{~Hz})$, theta wave $(4.0-8.0 \mathrm{~Hz})$, and the alpha wave $(8.0-13.0 \mathrm{~Hz})$. During the awake period, the coherence of slow waves $(<1.0 \mathrm{~Hz})$ was decreased. c The coherence spectra of the awake period and the period during induced LOC. The alpha wave (8.0-13.0 Hz) coherence is similar in both periods. However, the delta wave $(1.0-4.0 \mathrm{~Hz})$ during etomidate-induced LOC was more coherent than during the awake period (1.86-3.17 Hz, two-group test for coherence, $p<0.001$ ). Median coherence is presented with $95 \%$ jackknife Cls. Horizontal solid black lines represent frequency ranges at which there was significant difference. The bandpass filter allows frequency components to pass within a certain frequency range but attenuates the frequency components in other ranges to a very low level rather than cutting off frequencies outside the range at a certain frequency point; therefore, at $40 \mathrm{~Hz}$, the graph shows that the coherence is uniform and rising 
different frequency oscillations are related to changes in information coding and synaptic weights [27]. Etomidate-induced amnesia arises by means of GABAAR modulation, which highly depends on $\alpha-5$ subunit modulation; this process likely occurs principally within the hippocampus [28, 29]. Anesthetics may disturb the greatly organized rhythmic activity patterns that are thought to be indispensable for hippocampal learning [30]. For instance, changes in theta frequency and theta power may contribute to amnesia by means of anesthetic actions on a variety of molecular targets [31]. Theta rhythms act as internal clocks that synchronize large networks and serve as a reference mechanism for internal and external synchronization, which is greatly coherent throughout the medial temporal lobe. These findings suggest that etomidate-induced LOC may be related to the changes in hippocampal theta rhythms. Collectively, these potential associations have led us to hypothesize that etomidate-induced theta oscillations may be an indicator of a functional disconnection between the hippocampus and cerebral cortex.

The EEG data analyzed in this study were all derived from the frontal 4-channel pathway, so our analysis was unable to assess other reported cortical kinetic connectivity associated with anesthetic-induced LOC. The results have been obtained with frontal electrodes only as a study limitation, so our observations need to be further validated in future high-density EEG studies[32]. At present, exploring the relationship between the neural circuit mechanism of etomidate-induced LOC and the characteristic changes of EEG can only be carried out in animal experimental models. Therefore, the clinical observed 'the connection' still needs to be verified by animal models.

According to our analysis and discussion, from the awake period to the LOC, the neural circuit mechanism of etomidate-induced LOC is closely related to the enhancement of delta-wave, alpha-wave, and theta-wave coherence. The characteristic transformation of the EEG that we described can be calculated and displayed in real time, providing a good reference for the monitoring of the depth of anesthesia and the evaluation of the level of sedation and consciousness by an anesthesiologist.

\section{Conclusions}

The neural circuit mechanism of etomidate-induced LOC is closely related to the induction of oscillation in each EEG band and is closely related to the enhancement of delta-wave coherence. Through the analysis of EEG, our study elaborates the neural circuit mechanism related to the LOC induced by etomidate, which provides a feasible direction for subsequent clinical and basic studies.

\section{Abbreviations}

EEG: Electroencephalography; LOC: Loss of Consciousness; SEF95: Spectral Edge Frequency 95 \%; BIS: Bispectral Index; GABAAR: y-aminobutyric Acid A Receptor; ECG: Electrocardiography; ASA: American Society of

Anesthesiologists; LFPs: Local Field Potentials

\section{Authors' contributions}

LZ and SQF contributed equally and share first authorship with manuscript editing, and modifying figures and Tables. Other authors contributed to the writing, review and editing of the manuscript. All authors read and approved the final manuscript

\section{Funding}

Provincial Quality Engineering Project of Higher Education Institutions of Anhui Province (2017jyxm1080).

\section{Availability of data and materials}

All data generated or analyzed during this study are included in this published article.

\section{Declarations}

Ethics approval and consent to participate

This study followed the Declaration of Helsinki and was approved by the Ethics Committee of the First Affiliated Hospital of Anhui Medical University. Written informed consent was obtained from the patients, who were between 18 and 65 years old.

\section{Consent for publication}

Not Applicable.

\section{Competing interests}

The authors declare that they have no competing interests.

\section{Author details}

${ }^{1}$ Department of Anesthesiology, The First Affiliated Hospital of Anhui Medical University, No. 218 Jixi road, Anhui province 230022 Hefei, China. ${ }^{2}$ The First Medical College of Anhui Medical University, 230032 Hefei, China. ${ }^{3}$ Key Laboratory of Anesthesiology and Perioperative Medicine of Anhui Higher Education Institutes, Anhui Medical University, Hefei, China.

Received: 5 October 2020 Accepted: 25 February 2021

Published online: 08 April 2021

References

1. Fu B, Wang Y, Yang H, Yu T. Effects of Etomidate on GABAergic and Glutamatergic Transmission in Rat Thalamocortical Slices. Neurochemical Research. 2016:41:3181-91.

2. Pejo E, Santer P, Wang L, Dershwitz P, Husain SS, Raines DE. Y-Aminobutyric Acid Type A Receptor Modulation by Etomidate Analogs. Anesthesiology. 2016:124:651-63.

3. Gooding. JM, Weng. J-T, Smith. RA, Berninger. GT, Kirby. RR. Cardiovascular and Pulmonary, Responses following Etomidate Induction of Anesthesia in Patients with Demonstrated Cardiac Disease. Anesth Analg. 1979;58:40-1.

4. Hamme. MJV, M.M.Ghoneim., J.Ambre. J. Pharmacokinetics of Etomidate,a New Intravenous Anesthetic. Anesthesiology. 1978;49:274-7.

5. Alkire. MT, Hudetz. AG, Tononi. G. Consciousness and Anesthesia. Science. 2008;322:876-80.

6. Franks NP. General anaesthesia: from molecular targets to neuronal pathways of sleep and arousal. Nat Rev Neurosci. 2008;9:370-86.

7. Isley MR, Edmonds HL, Jr., Stecker M, American Society of Neurophysiological M. Guidelines for intraoperative neuromonitoring using raw (analog or digital waveforms) and quantitative electroencephalography: a position statement by the American Society of Neurophysiological Monitoring. J Clin Monit Comput. 2009;23:369-90.

8. Serfontein L. Awareness in cardiac anesthesia. Curr Opin Anaesthesiol. 2010; 23:103-8.

9. Weiner VS. Intracranial Electroencephalography Signatures of the Induction of General Anesthesia with Propofol. Massachusetts Institute of Technology. 2013. 
10. Kuizenga K, Wierda JMKH, Kalkman CJ. Biphasic EEG changes in relation to loss of consciousness during induction with thiopental, propofol, etomidate, midazolam or sevoflurane. British Journal of Anaesthesia. 2001;86:354-60.

11. Huter L, Schreiber T, Gugel M, Schwarzkopf K. Low-dose intravenous midazolam reduces etomidate-induced myoclonus: a prospective, randomized study in patients undergoing elective cardioversion. Anesth Analg. 2007;105:1298 - 302, table of contents.

12. Yates $A M$, Wolfson $A B$, Shum $L$, Kehrl T. A descriptive study of myoclonus associated with etomidate procedural sedation in the ED. Am J Emerg Med. 2013;31:852-4

13. A.Kearse. L, Manberg. P, Chamoun. N, deBros. F, Zaslavsky. A. Bispectral Analysis of the Electroencephalogram Correlates with Patient Movement to Skin Incison during Propofol/Nitrous Oxide Anesthesia. Anesthesiology. 1994;81:1365-70.

14. Malekmohammadi M, Price CM, Hudson AE, DiCesare JAT, Pouratian N. Propofol-induced loss of consciousness is associated with a decrease in thalamocortical connectivity in humans. Brain. 2019;142:2288-302.

15. Pierre MS, Landsleitner B, Schwilden H, Schuettler J. Awareness During Laryngoscopy and Intubation: Quantitating Incidence Following Induction of Balanced Anesthesia With Etomidate and Cisatracurium as Detected With the Isolated Forearm Technique. Journal of Clinical Anesthesia. 2000;12:104-8.

16. DB P, AT W. Spectral Analysis for Physical Applications. New York: Cambridge University Press. 1993.

17. Akeju. O, Westover. MB, Pavone. KJ, Sampson. AL, Hartnack. KE, Brown. EN,et al. Effects of Sevoflurane and Propofol on Frontal Electroencephalogram Power and Coherence. Anesthesiology. 2014;121:990-8.

18. Bokil H, Purpura K, Schoffelen JM, Thomson D, Mitra P. Comparing spectra and coherences for groups of unequal size. J Neurosci Methods. 2007;159:337-45.

19. Jurd R, Arras M, Lambert S, Drexler B, Siegwart R, Crestani F,et al. General anesthetic actions in vivo strongly attenuated by a point mutation in the GABAA receptor $\beta 3$ subunit. The FASEB Journal 2002.

20. O'Meara GF, Newman RJ, Fradley RL, Dawson GR, Reynolds DS. The GABA-A beta3 subunit mediates anaesthesia induced by etomidate. Neuroreport. 2004;15:1653-6.

21. Drexler B, Roether CL, Jurd R, Rudolph U, Antkowiak B. Opposing Actions of Etomidate on Cortical Theta Oscillations Are Mediated by Different $\gamma$ Aminobutyric Acid Type A Receptor Subtypes. Anesthesiology. 2005;102:346-52.

22. Butovas S, Rudolph U, Jurd R, Schwarz C, Antkowiak B. Activity Patterns in the Prefrontal Cortex and Hippocampus during and after Awakening from Etomidate Anesthesia. Anesthesiology. 2010;113:48-57.

23. Hyman JM, Zilli EA, Paley AM, Hasselmo ME. Working Memory Performance Correlates with Prefrontal-Hippocampal Theta Interactions but not with Prefrontal Neuron Firing Rates. Front Integr Neurosci. 2010;4:2.

24. Siapas AG, Lubenov EV, Wilson MA. Prefrontal Phase Locking to Hippocampal Theta Oscillations. Neuron. 2005;46:141-51.

25. Maciver MB, Roth SH. Inhalation Anaesthetics Exhibit Pathwayspecific And Differential Actions On Hippocampal Synaptic Responses In Vitro. Br J Anaesth. 1988:60:680-91.

26. Nishikawa K-i, Maclver MB. Excitatory Synaptic Transmission Mediated by NMDA Receptors Is More Sensitive to Isoflurane than Are Non-NMDA Receptor-mediated Responses. Anesthesiology. 2000;92:228-36.

27. Buzsaki G, Moser El. Memory, navigation and theta rhythm in the hippocampal-entorhinal system. Nat Neurosci. 2013;16:130-8.

28. Belelli D, Lambert JJ, Peters JA, Wafford K, Whiting PJ. The interaction of the general anesthetic etomidate with the $\gamma$-aminobutyric acid type A receptor is influenced by a single amino acid. Proc Natl Acad Sci. 1997;94:11031-6.

29. Cheng VY, Martin LJ, Elliott EM, Kim JH, Mount HT, Taverna FA,et al. Alpha5GABAA receptors mediate the amnestic but not sedative-hypnotic effects of the general anesthetic etomidate. J Neurosci. 2006:26:3713-20.

30. Perouansky M, Pearce RA. How we recall (or don't): the hippocampal memory machine and anesthetic amnesia. Can J Anaesth. 2011:58:157-66.

31. Perouansky M, Hentschke H, Perkins M, Pearce RA. Amnesic Concentrations of the Nonimmobilizer 1,2-Dichlorohexafluorocyclobutane (F6, $2 \mathrm{~N}$ ) and Isoflurane Alter Hippocampal $\theta$ Oscillations In Vivo. Anesthesiology 2007;106:1168-76.

32. Lee U, Ku S, Noh G, Baek S, Choi B, Mashour GA. Disruption of FrontalParietal Communication by Ketamine, Propofol, and Sevoflurane. Anesthesiology. 2013;118:1264-75.

\section{Publisher's Note}

Springer Nature remains neutral with regard to jurisdictional claims in published maps and institutional affiliations.

Ready to submit your research? Choose BMC and benefit from:

- fast, convenient online submission

- thorough peer review by experienced researchers in your field

- rapid publication on acceptance

- support for research data, including large and complex data types

- gold Open Access which fosters wider collaboration and increased citations

- maximum visibility for your research: over $100 \mathrm{M}$ website views per year

At BMC, research is always in progress.

Learn more biomedcentral.com/submissions 